\title{
Research Paper \\ Impact of Psychosocial Conditions on the Public Services Motivation of Employees of Qazvin University of Medical Sciences
}

\section{Seyede Saeede Javanmard ${ }^{1}$ (), Nasrin Jazani ${ }^{2 *}$ (), Seyed Mehdi Alvani ${ }^{3}$, Mohammad Reza Hamidizadeh ${ }^{2}$}

1. Department of Management, Faculty of Management, Islamic Azad University of Qazvin, Qazvin, Iran.

2. Department of Management, Faculty of Management and Accounting, Shahid Beheshti University, Tehran, Iran.

3. Department of Management, Faculty of Management and Accounting, Allameh Tabataba'i University, Tehran, Iran.

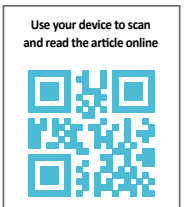

Citation Javanmard SS, Jazani N, Alvani SM, Hamidizadeh MR. Impact of Psychosocial Conditions on the Public Services Motivation of Employees of Qazvin University of Medical Sciences. The Journal of Qazvin University of Medical Sciences. 2019; 23(1):62-73. https:// doi.org/10.32598/JQUMS.23.1.62

dol https://doi.org/10.32598/JQUMS.23.1.62

Received: 08 Aug 2018

Accepted: 26 Nov 2018 Available Online: 01 Apr 2019

Keywords:

Motivation, Psychological conditions, Environmental factors

\section{A BSTRACT}

Background Many scholars agree that one of the most important dimensions of public sector is the Public Service Motivation (PSM) of employees.

Objective We investigated the psychological aspects associated with occupational and organizational factors, as well as the moderating role of the environment on PSM from the viewpoint of employees of Qazvin University of Medical Sciences.

Methods This descriptive cross-sectional study was conducted between 180 employees of Qazvin University of Medical Sciences in 2018. Kim and Vandenabeele, Hackman and Oldham's Job Diagnostic Survey, and two researcher-made questionnaires were used in the current research. All statistical tests were performed at the error level of 0.05 . Structural equations with partial least squares method have also been used.

Findings The psychological aspect of profession has the most significant impact on the employee's job motivation. Moreover, the feeling of satisfaction has the strongest association with the operation of public interest; and knowledge of the results of the least effective. In the case of variables affecting the psychological condition of the organization, the individual's perception about the spatial design of the organization, the most and most variable of the individual's feelings of membership in the organization has the least effect.

Conclusion Human Resource Management is the most important task of managers, and to increase the effectiveness of government agencies performance, the human resources motivation system should be improved.

\section{Extended Abstract}

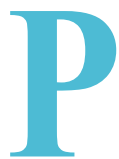

\section{Introduction}

romoting employee's motivation has always been a major concern for managers. Today's managers should understand why their employees work and appropriately re- ward them [2]. In this regard, it is beneficial to investigate the factors that motivate the employees of the public sector

Critical psychological states form the core of Hackman and Oldham's theory (1980). According to them, these states show how people respond to their responsibilities under the influence of occupational characteristics [15]. They divide critical psychological states into three categories:

\section{* Corresponding Author}

Nasrin Jazani

Address: Department of Management, Faculty of Fanagement and Accounting, Shahid Beheshti University, Tehran, Iran.

Tel: +98 (912) 1300295

E-Mail: nasrin_jazani@yahoo.com 
experienced meaningfulness, experienced responsibility for outcomes, and knowledge of the actual results. The Social Information Processing (SIP) model was developed by Salancik and Pfeffer (1977).

In this model, psychological states based on organizational factors are: 1. Employee's sense of membership in the organization (choice); 2.Employee's sense of leaving the organization (revocability); 3. Employee's sense of the organization's visibility (publicness) [5]. This study aimed to identify and explain critical psychological states caused by occupational (Hackman and Oldham's theory) and organizational factors (Salancik and Pfeffer theory) that affect Public Services Motivation (PSM) of employees by considering environmental factors (including economic and geographical environment) as a moderating variable in the study organization. The research questions are: 1. What model can we use to create PSM?; 2. What are the constructs of the PSM model?; 3. What are the dimensions/ components of the constructs of the PSM model?; 4. What is the effect of moderating environmental factors for the effectiveness of job-related psychological states on PSM?; 5. What is the effect of moderating environmental factors for the effectiveness of organization-related psychological states on PSM?

\section{Materials and Methods}

This research is a descriptive survey. The statistical population consisted of official and contract employees of Qazvin University of Medical Sciences $(n=343)$. Of these, 180 samples were selected using stratified random sampling technique and Krejcie and Morgan table. To assess PSM in the samples, we used Kim and Vandenabeele questionnaire (2010). The occupational factors were adapted from the Job Diagnostic Survey (JDS) tool of Hackman and Oldham (1974), which assesses the satisfaction with public interest practice.

Besides, two researcher-made questionnaires were used for surveying organizational and environmental factors.
To answer the research questions, we performed the Structural Equation Modeling (SEM) with Partial Least Squares (PLS) approach in Smart PLS software. The Cronbach $\alpha$ coefficient for the questionnaire assessing psychological states caused by occupational factors was obtained as 0.83 . Also, the $\alpha$ coefficient for the questionnaire assessing psychological states caused by organizational factors was calculated as 0.85 . Finally, the $\alpha$ coefficient for the questionnaire assessing psychological states caused by environmental factors was found as 0.86 .

\section{Results}

For evaluating Goodness-Of-Fit (GOF) of the measurement model, we used the parameters of reliability index, content validity, convergent validity, and divergent validity. For assessing content validity, several academic experts examined and monitored the accuracy and clarity of the items. Table 1 shows the results of convergent validity, divergent validity, and coefficient of determination.

In the next step, to examine the effect of moderating variable, we entered the environmental factors into the measurement model. Since only one variable can be dependent on each step, we measured the effect of environmental factors in two steps: 1. Examining their effect in moderating the relationship of PSM with psychological states caused by occupational factors; 2 . Examining their effect in moderating the relationship of PSM with psychological states caused by organizational factors. To prioritize the impact of each dimension of environmental factors, we introduced these dimensions separately and individually as a modifying variable to the software. Table 2 presents the results.

\section{Discussion}

"Experienced meaningfulness" had the highest effect and "knowledge of the actual results" had the lowest impact on having psychological states caused by occupational factors. Therefore, employees will experience higher PSM in fulfilling their duties, if they take into account the purpose of

Table 1. Convergent validity, divergent validity, and coefficient of determination

\begin{tabular}{|c|c|c|c|c|c|c|c|}
\hline Variables & 1 & 2 & 3 & $\begin{array}{l}\text { The Cronbach } \alpha \\
\qquad>0.7)\end{array}$ & $\begin{array}{l}\text { The Combined Reli- } \\
\text { ability }(>0.7)\end{array}$ & $\begin{array}{l}\text { The Average Variance } \\
\text { Extracted (AVE) }\end{array}$ & $\mathbf{R}^{2}$ \\
\hline PSM & 0.63 & & & & & & \\
\hline $\begin{array}{l}\text { Psychological states caused by } \\
\text { occupational factors }\end{array}$ & 0.72 & 0.96 & & 0.87 & 0.91 & 0.67 & 0.52 \\
\hline $\begin{array}{l}\text { Psychological states caused } \\
\text { by organizational factors }\end{array}$ & 0.48 & 0.45 & 0.65 & 0.71 & 0.74 & 0.42 & 0.43 \\
\hline
\end{tabular}


Table 2. Prioritizing the environmental factors based on their moderating effect

\begin{tabular}{cccccccc}
\hline & Moderator & & Factor Loads & Sig. & $\mathbf{R}^{2}$ & Effect Size & Rank \\
\hline $\begin{array}{c}\text { Organizational } \\
\text { factors }\end{array}$ & $\begin{array}{c}\text { Environmental } \\
\text { factors }\end{array}$ & $\begin{array}{c}\text { Economic } \\
\text { environment } \\
\text { Geographical } \\
\text { environment }\end{array}$ & 0.435 & 2.157 & 0.986686 & 0.089 & 1 \\
$\begin{array}{c}\text { Occupational } \\
\text { factors }\end{array}$ & $\begin{array}{c}\text { Environmental } \\
\text { factors }\end{array}$ & $\begin{array}{c}\text { Geographical } \\
\text { environment } \\
\text { Economic } \\
\text { environment }\end{array}$ & 0.315 & 1.964 & 0.981183 & 0.083 & 2 \\
\hline & & 0.326 & 1.972 & 0.981179 & 0.077 & 2 \\
\hline
\end{tabular}

serving and helping others [16]. "sense of the visibility" and "sense of membership in the organization" had the highest and lowest effect on having psychological states caused by organizational factors, respectively. Hence, when employees are allowed to speak clearly about the work concerns or problems in the organization, they can experience a higher level of PSM [19].

The moderating effect of environmental factors was higher regarding the relationship of PSM with psychological states caused by organizational factors than when it moderates the relationship of PSM with job-related psychological states. Therefore, the research findings indicate that timely payment of salaries, appropriate benefits, the proportionality of pay rise with the inflation rate, and the existence of suitable and stable economic condition are predictors of PSM. In addition, the increasing gap between the rich and the poor will cause distress for employees [12].

\section{Ethical Considerations}

\section{Compliance with ethical guidelines}

All ethical principles were considered in this article. The participants were informed about the purpose of the research and its implementation stages; they were also assured about the confidentiality of their information; Moreover, They were allowed to leave the study whenever they wish, and if desired, the results of the research would be available to them.

\section{Funding}

This paper was extracted from a $\mathrm{PhD}$ thesis of the first author, Department of Public Managment, University of 1slamic Azad University of Qazvin in governmental management approved by Islamic Azad University of Qazvin Branch (Code: 21421210941078).

\section{Authors' contributions}

Study conceptualization, methodology, validation, formal analysis, resources, data curation, editing, supervision, project administration and funding acquisition: Seyed Saeede Javanmard; Methodology, validation, formal analysis, supervision, and project administration: Nasrin Jazani, Seyed Mehdi Alvani, and Mohamad Reza Hamidizadeh.

\section{Conflicts of interest}

The authors declared no conflict of interest. 


\title{
تأثير حالات روانى حساس بر انكَيزش خدمات عمومى كاركنان دانشَّاه علوم يزشكى قزوين
}

\author{
سيده سعيده جوانمرد' هـ، "نسرين جزنى ' ه، سيد مهدى الوانى"، محمدرضا حميدىزاده
}

ا- كروه هديريت دولتى، دانشكده مديريت، واحد قزوين، دانشكاه آزاد اسلامى، قزوين، ايران.

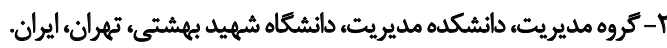

r- كروه مديريت، دانشكده مديريت و حسابدارى، دانشكاه علامه طباطبائي، تهران، ايران.

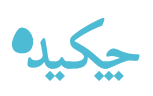

إنمينه بسيارى ازو يُؤهشكران توافق دارند كه امروزه يكى أز مهمترين و رايج ترين ابعاد در بخش عمومى، انكيزش كاركنان بخش عمومى

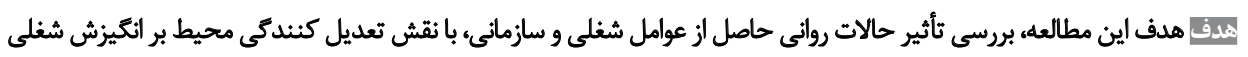

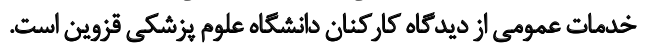

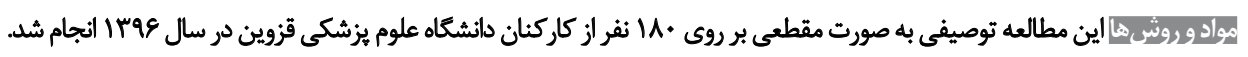

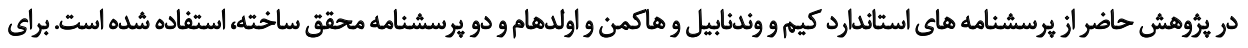

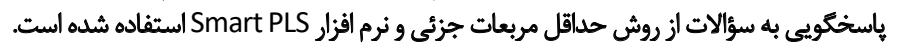

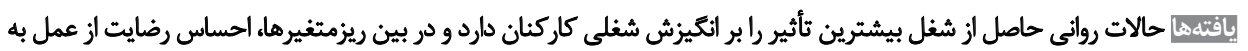

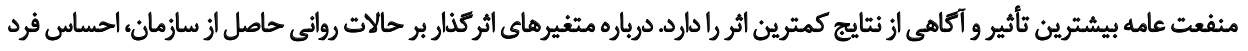

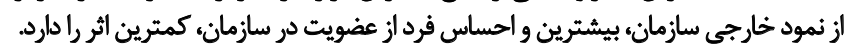

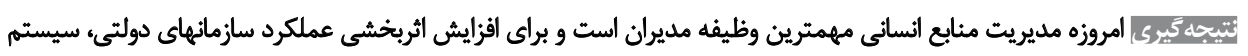
أنكيزشى مثابع أسانى بايد بهبود يابد.
\end{abstract}

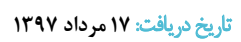

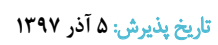

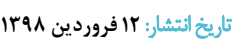

كليسوازوهاء

انتيزش، حالات روانى حساس، عوامل محيطى نانى
جه عواملى كاركنان بخش دولتى را برمىانگيزد، بسيار مفيد

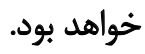

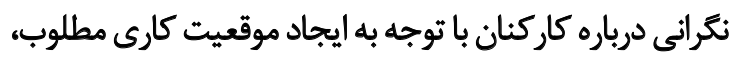

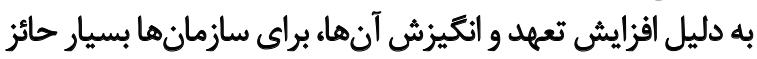

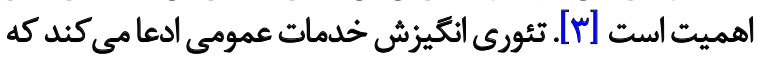

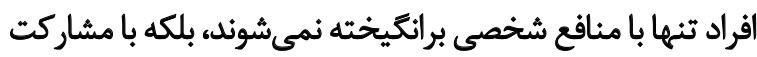

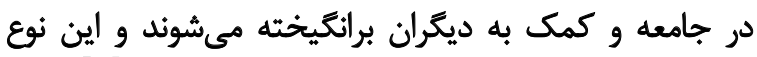

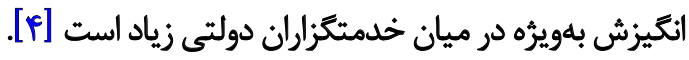

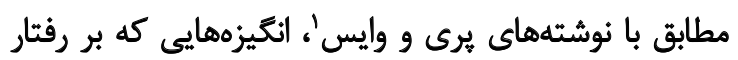

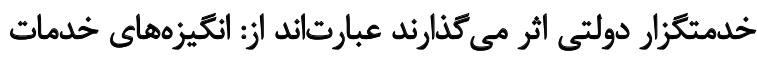

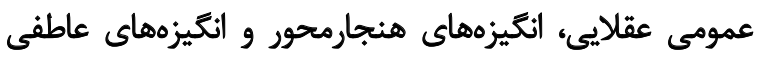

\section{Perry \& Wise}

dale

انكيزش فرايندى است كه تحريك، هدايت و حمايت از تلاش

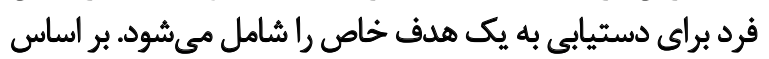

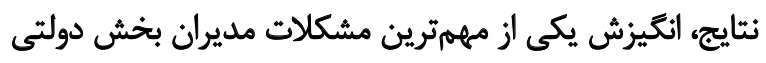

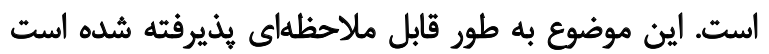

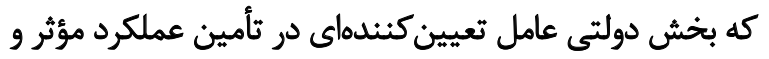

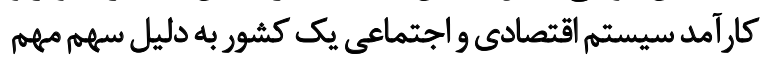

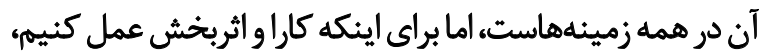

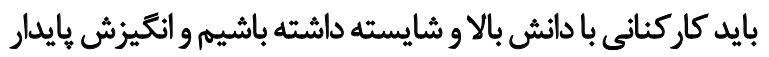
كاركنان را حفظ كنيم [1]

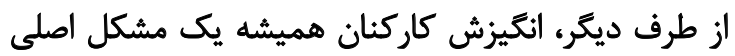

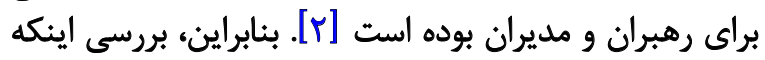




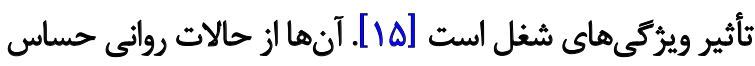

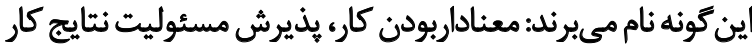

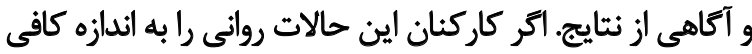

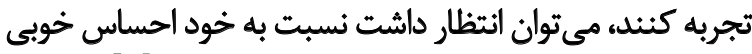

داشته و برخورد مناسبى با شغل خود داشته باشند [هان]

در يُروهش حاضر، برداشتى احساسى از مدل برورش اطلاعات

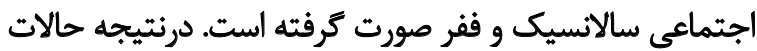

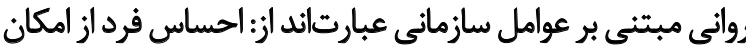

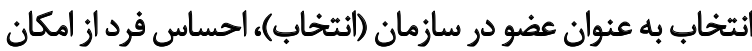

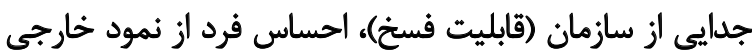

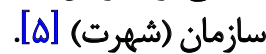

از اين رو، هدف بُروهش حاضر، شناخت و تبيين حالات

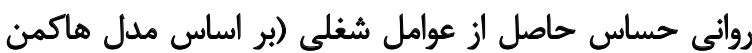

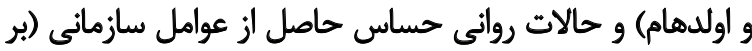

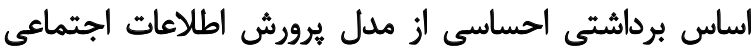

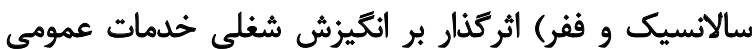

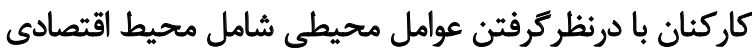
(رونق و ركود) و جغرافيايى به عنوان متغير تعديلكر در سازمان ماني

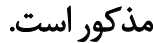

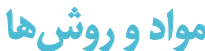

يثروهش حاضر به لحاظ هدف كاربردى است، زيرا به دنبال ارائه

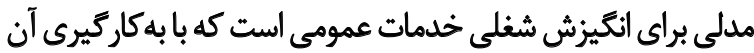

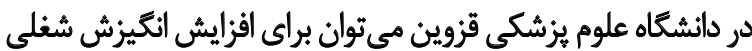

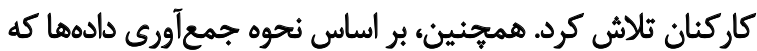

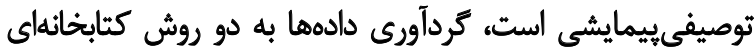

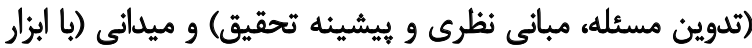

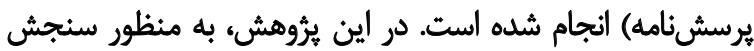

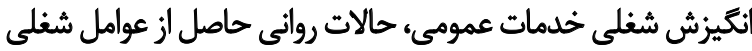

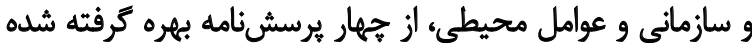

در هر جهار يرسشنامه از مقياس بنجزينهائ ليكرت استفاده

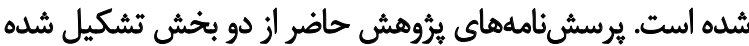

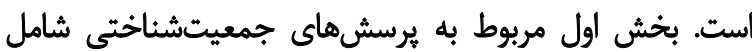

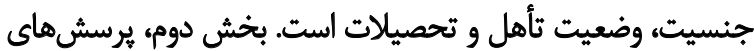

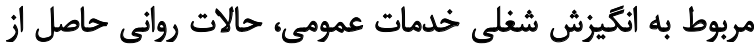

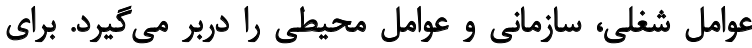

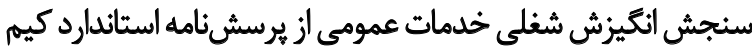

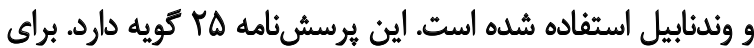

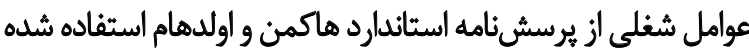

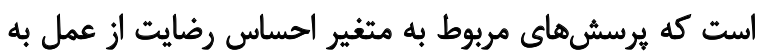
منفعت عامه به آن اضافه شده است.

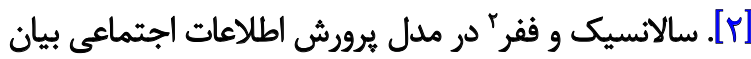

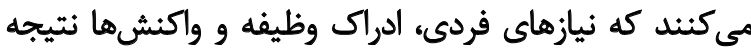

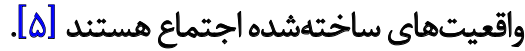

از طرف ديكر، بى تفاوتى كاركنان نسبت به مشكلات و مسائل

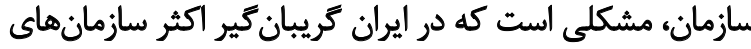

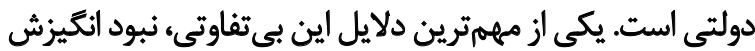

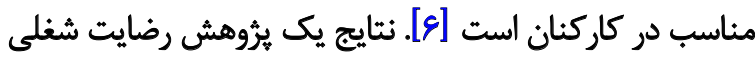

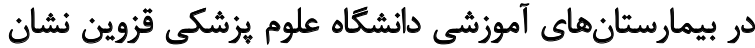

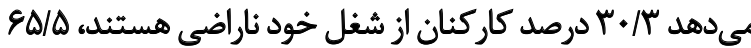

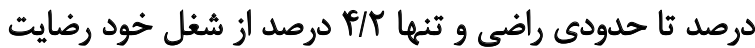

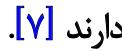

انكيزش، تمايل به انجام تلاش زياد براى اهداف سازمانى تعريف

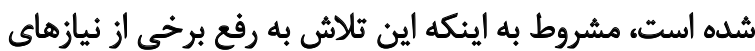

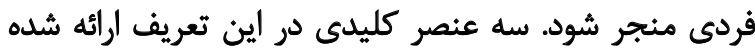

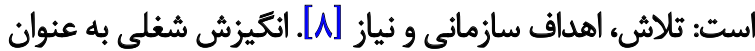

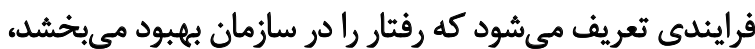

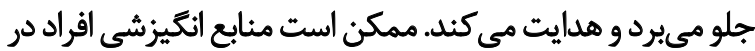

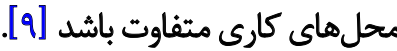

وندنبيل"، انغَيزش خدمات عمومى رابه عنوان باورها، ارزشهاو

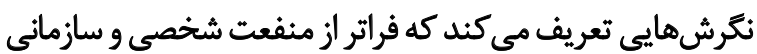

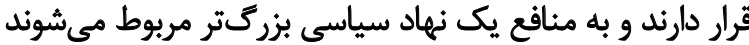

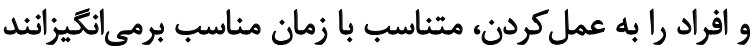

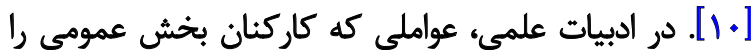

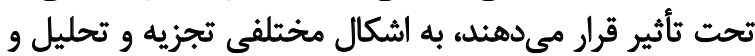

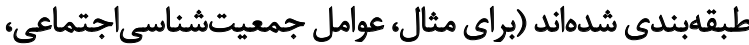

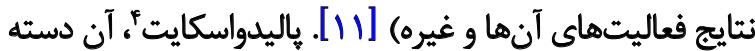

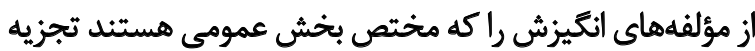

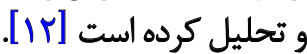

در يرُوهش ديكّر، در بين عوامل انكَيزشى درونى، اين عوامل

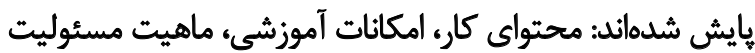

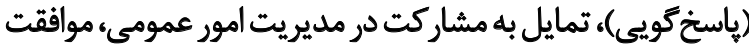

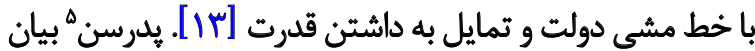

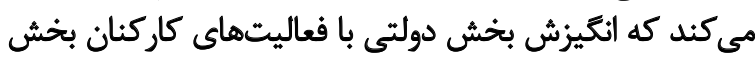

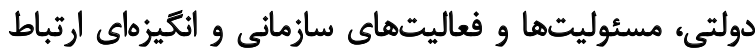

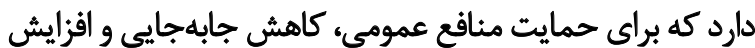
رضايت شغلى تلاش مى كنيند [If]

هاكمن و اولدهام" بيان ميكنيند كه حالات روانى حساس

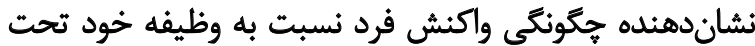

2. Salancik \& Pfeffer

3. Vandenabeele

4. Palidauskaité

5. Pedersen

6. Hackman \& Oldham 


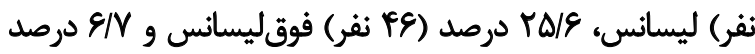

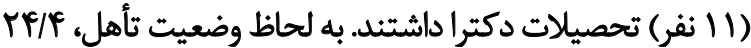

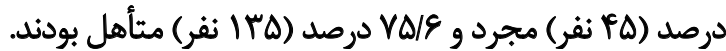

براى آزمون مدل يرّوهش، از معادلات ساختارى به روشي

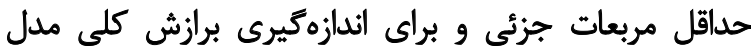

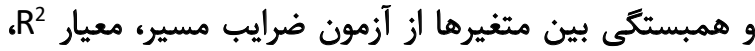

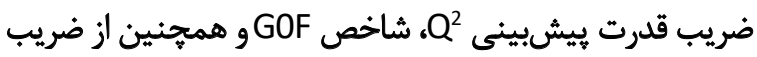

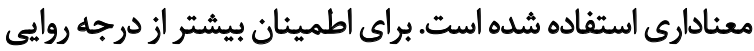

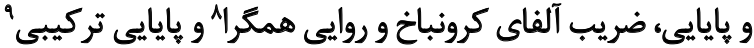

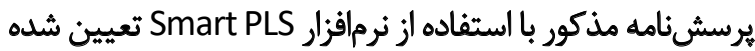

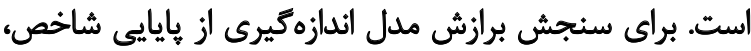

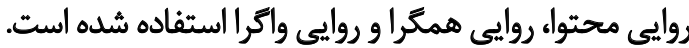

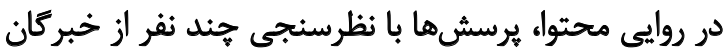

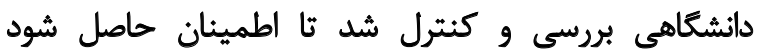

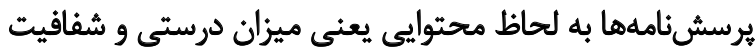

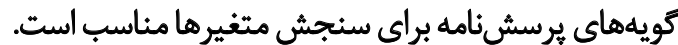

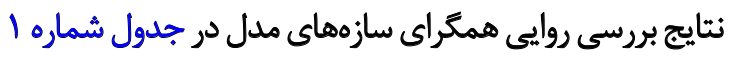

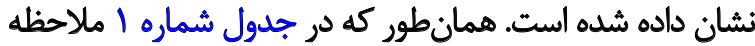

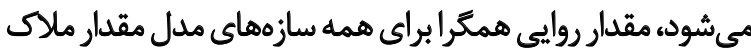

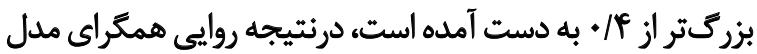

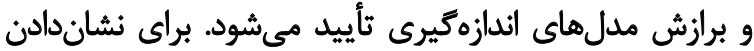

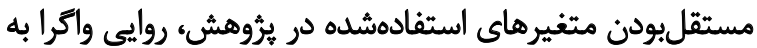

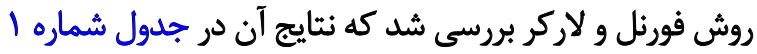

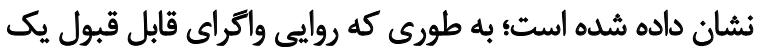

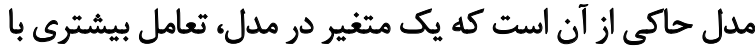
شاخصهاى خود دارد تا با متغيرهاى ديكي ميكر. با توجه به جدول شماره ا، مقدار جذر ميانكين واريانس

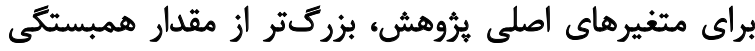

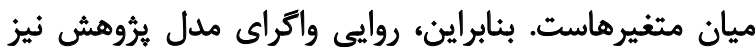

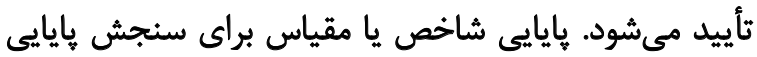

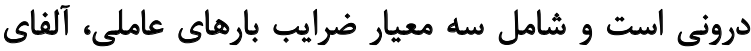

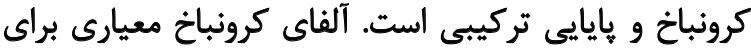

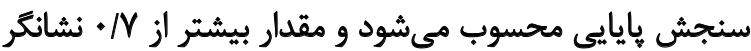
هايايى قابل قبول است.

مطابق باجدول شماره ا، همه عامل هاضريب يايايى قابل قبولى

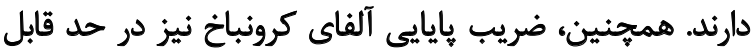

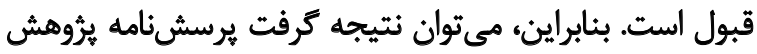

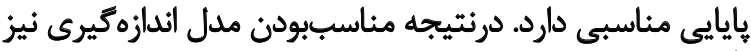

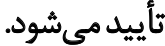

8. Average Variance Extracted (AVE)

9. Composite Reliability (CR)

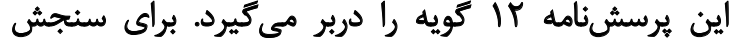

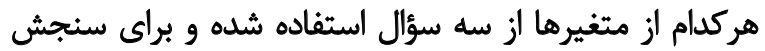

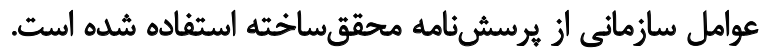

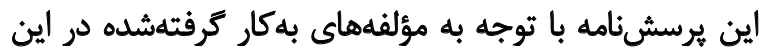

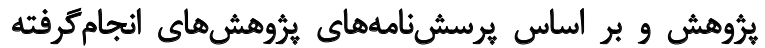

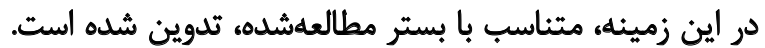

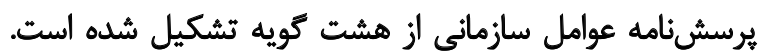

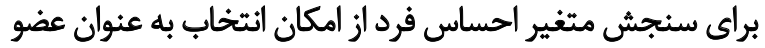

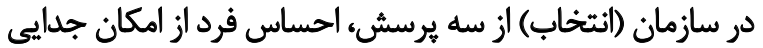

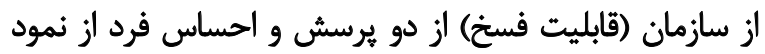

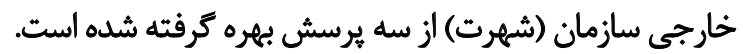

به منظور سنجش عوامل محيطى برسشنامهاى شامل 11

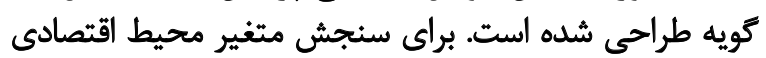

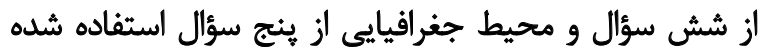

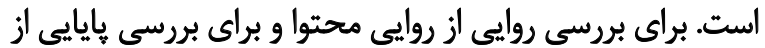

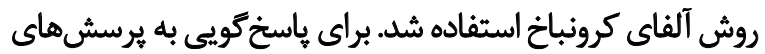

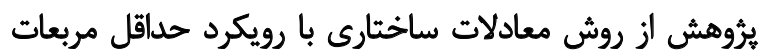

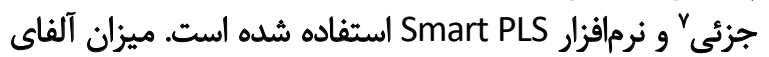

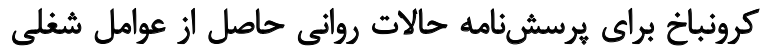

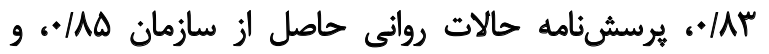

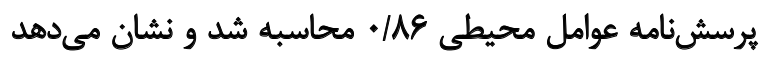
كه برسشنامهها يايايى خوبى دارند.

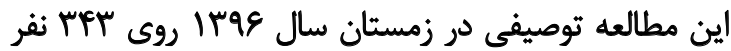

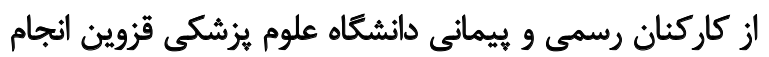

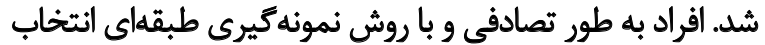

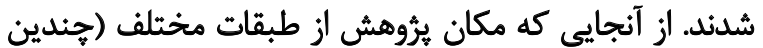

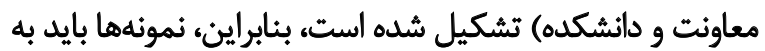

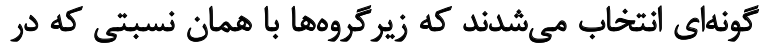

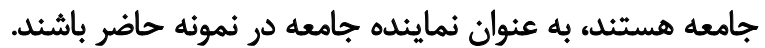

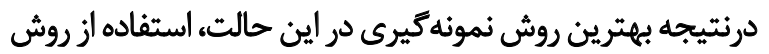

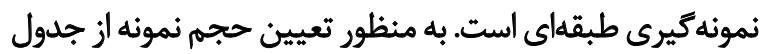

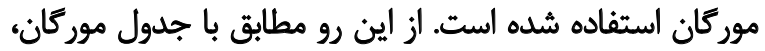

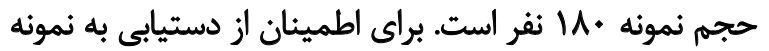

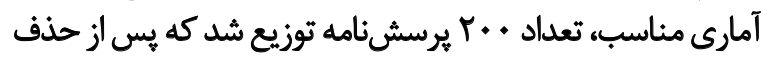

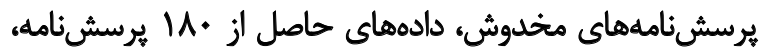
تجزيه و تحليل شد.

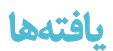

نتايج تحليل توصيفى دادههاى حاصل از يرسش نامنامه نشان

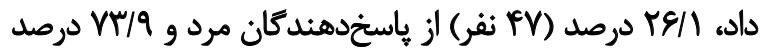

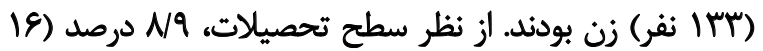

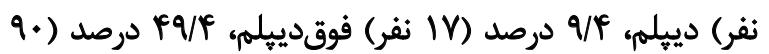
7.Partial Least Squares (PLS) 
ابعاد به طور جداكائه و تكتك به عنوان متغير تعديلكُ به نرمافزار

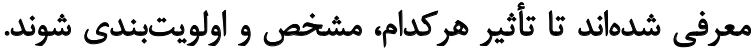

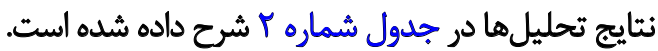
شدت اثر بهدست آمده براى تمامى ابعاد متغير تعديلكر عوامل

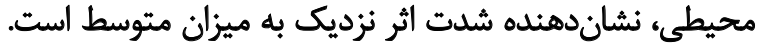

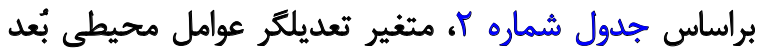

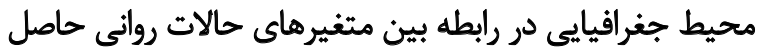

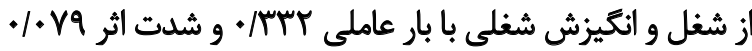

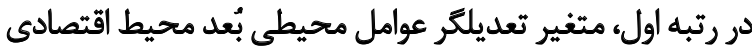

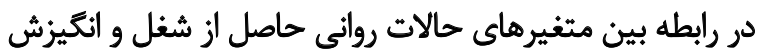

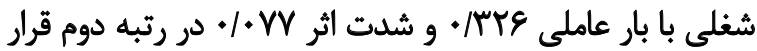

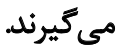

مرحله دوم) بررسى اثر متغير تعديلكر عوامل محيطى در رابطه

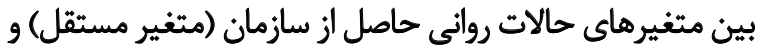
انكيزش شغلى خدمات عمومى (متغير وابسته).

ضريب معنادارى مربوط به متغير تعديل كننده (عوامل محيطى)

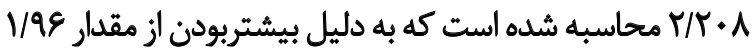

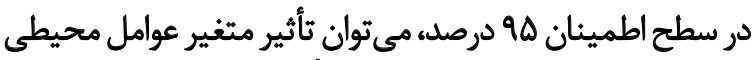

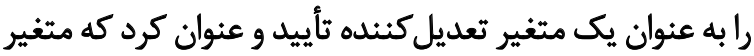

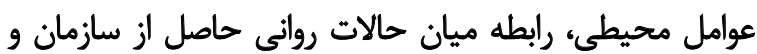
انكيزش شغلى خدمات عمومى را تعديل مي كئد.

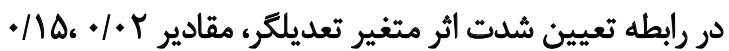

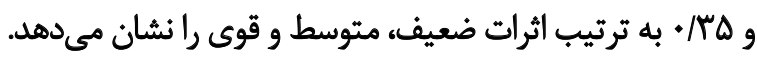

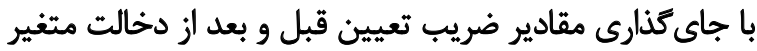

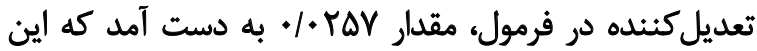

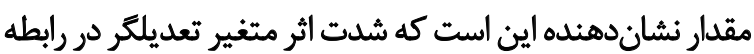

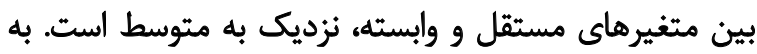

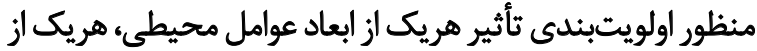

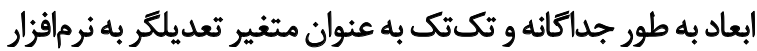

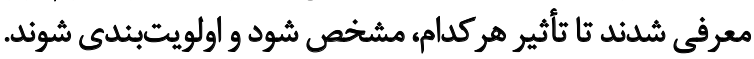
نتايج تحليل ها در جدول شماره ب شرح دادئ شاده شده است.

شدت اثر بهدستآمده براي تمامى ابعاد متغير تعديلكر عوامل

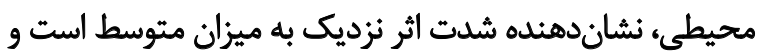

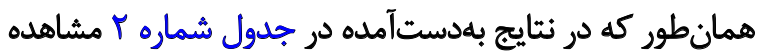

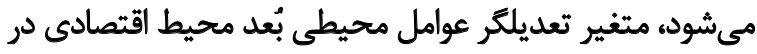

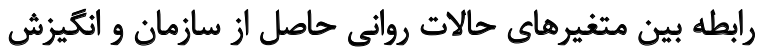

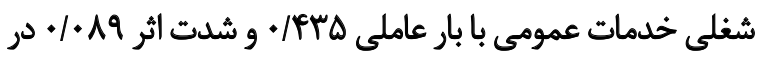

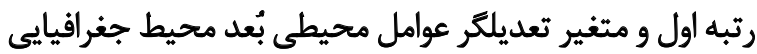

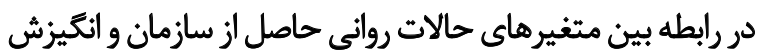

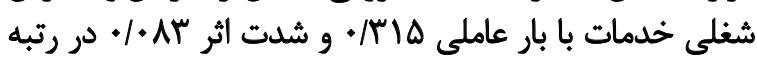

$$
\text { دوم قرار مي خيرند. }
$$

درنهايت بارهاى عاملى بررسى شد. مطابق با الكوريتم تحليل

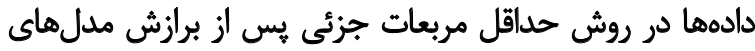

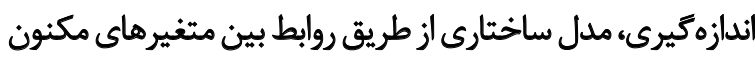

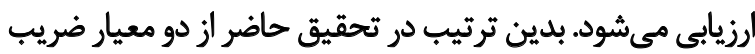
معنادارى " و ضريب تعيين (R²) استفاده شده است.

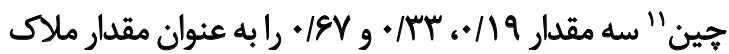

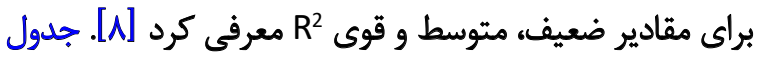

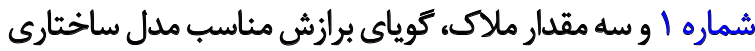
است.

درنهايت براى برازش كلى مدل از معيار GOF استفاده شده

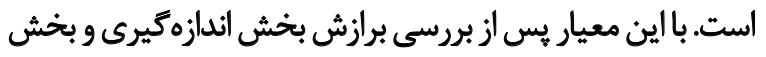

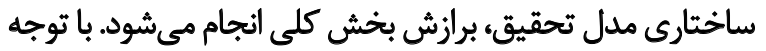

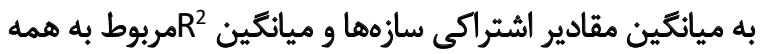

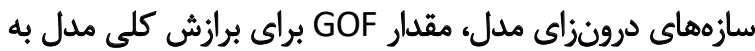

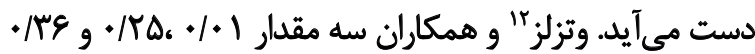

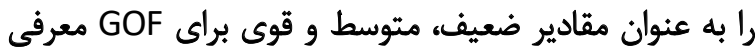

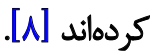

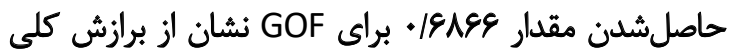

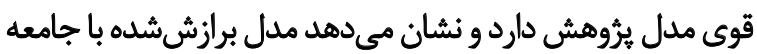

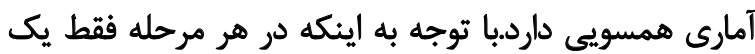

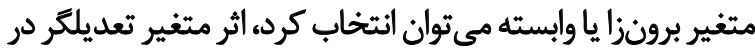
دو مر حله سنجيده مي ئشود. مرحله اول) بررسى اثر متغير تعديلكَ عوامل محيطى در رابطه

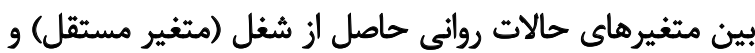
انغيزئش شغلى خدمات عمومى (متغير وابسته).

ضريب معنادارى مربوط به متغير تعديلكنينده (عوامل

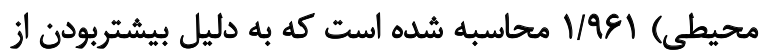

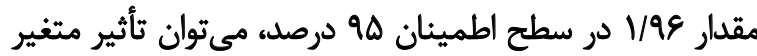

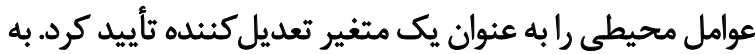

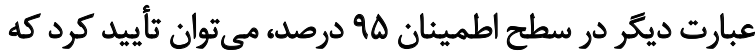

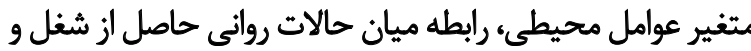
انكَيزش شغلى خدمات عمومى را تعديل كند.

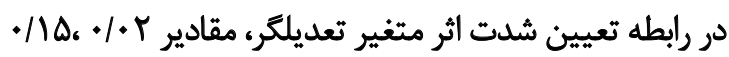

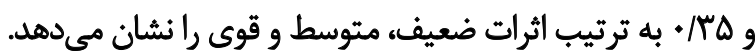

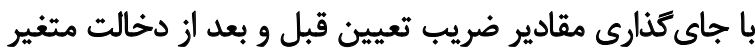

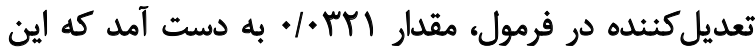

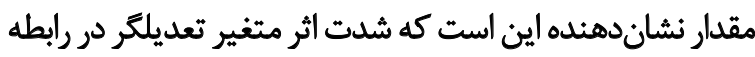

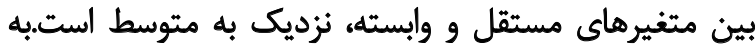

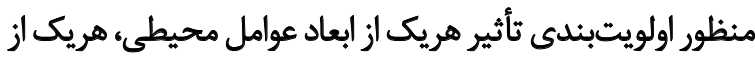

\section{T-Values}

11. Chein

12. Votzelz 


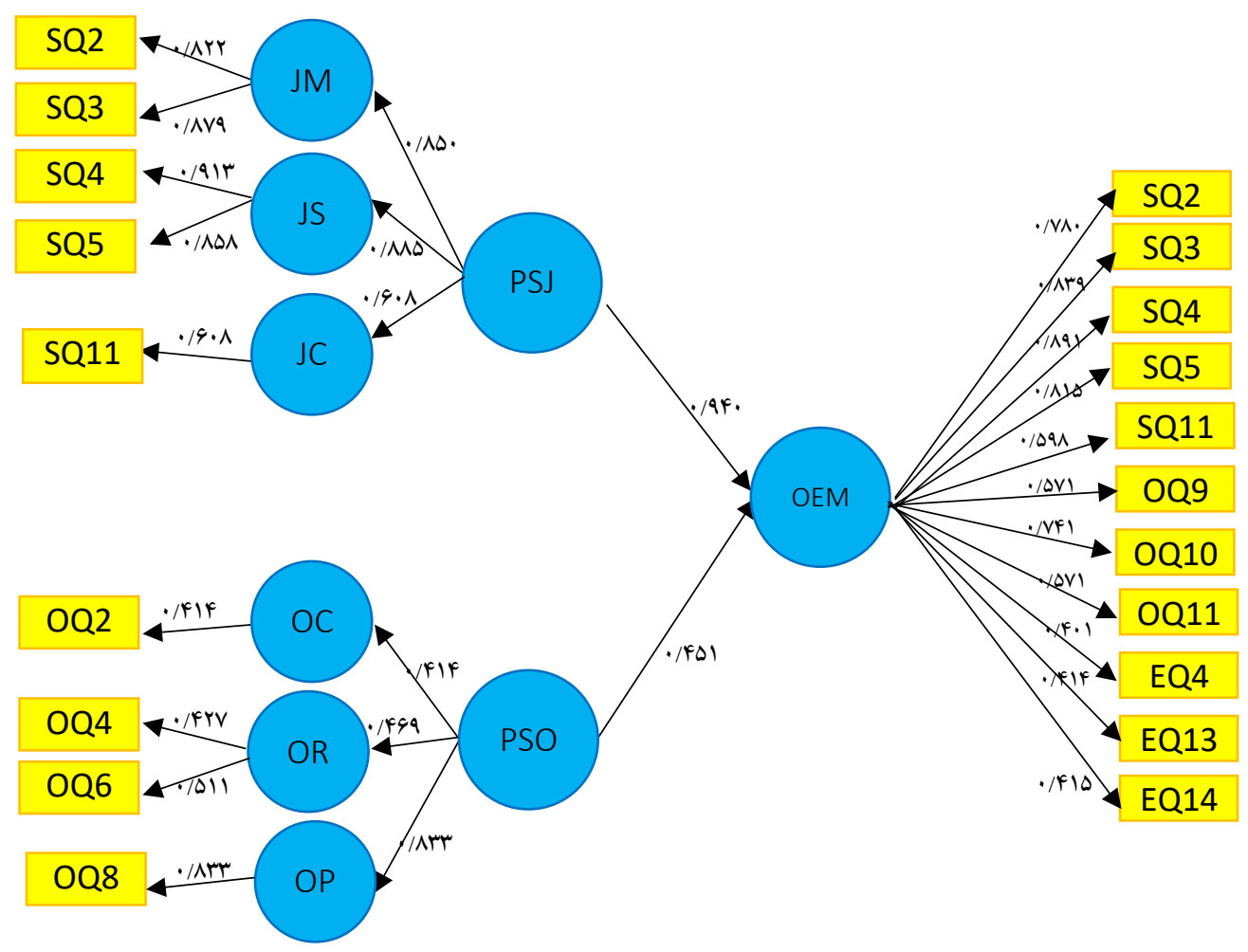

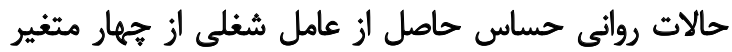

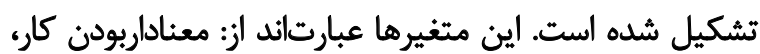

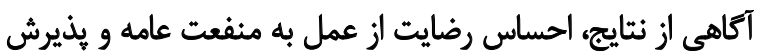

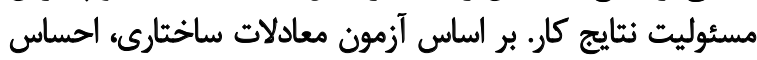

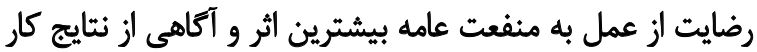

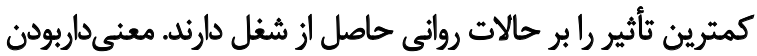

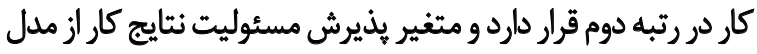

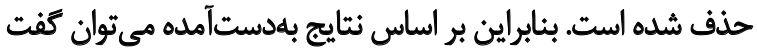

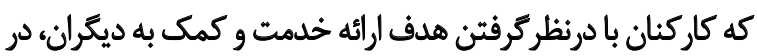

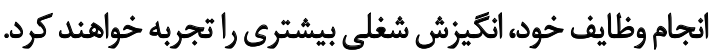
اين نكته كه افراد در سازمانهاى دولتي، ارائه خدمث به ديكران

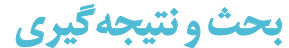

بر اساس مدل ارائهشده در اين برؤهش، حالات روانى اثركذار

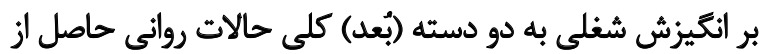

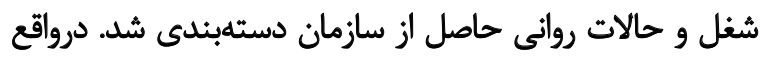

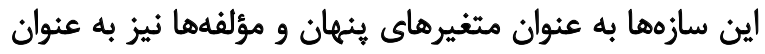

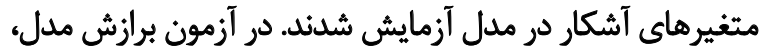

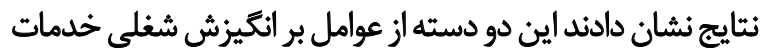

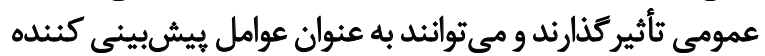

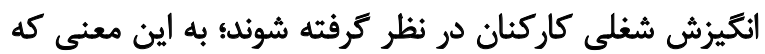

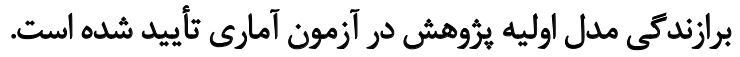

جدول ال. رواييى همكرا، واكرا و ضرايب خايايى مدل و ضريب تعيين

\begin{tabular}{|c|c|c|c|c|c|c|c|}
\hline $\mathbf{R}^{2}$ & AVE & بإيايى تركيبي(>/N) & ألفاي كرونباخ(|/> (>) & 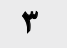 & $r$ & 1 & متغيرها \\
\hline & & & & & &.$/ F \pi$ & أثكيزش خلثات عمومى \\
\hline - /ar &.$/ E V$ & .191 & - MAY & &.$/ 9$ & $M$ & جاصل از شئل \\
\hline.$/ M$ & . $/ 4$ & $\cdot M^{\circ}$ & 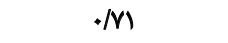 &.$/ 8 \Delta$ &.$/ 10$ &.$/ 14$ & حاصل از سازمان \\
\hline
\end{tabular}


جدول r. ابعاد عوامل محيطي در رابطه بين متغير هاى حالات روانى حاصل از شغل و سازمان با انكيزش خدمات عمومي

\begin{tabular}{|c|c|c|c|c|c|c|c|}
\hline رتبهبثدى متغيرهاى تعديلكر & شدث اثر متغير تعديلكر & $\mathbf{R}^{2}$ & ضرايب معنادارى & بارهاى عاملى & & متغير تعديلكر & \\
\hline رتبه اول & $.1 \times 19$ & - Rusen & $Y / \backslash Q Y$ & . & محيط اقتصادى & & \\
\hline رتبه دوم & 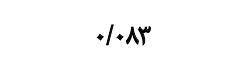 & ./MAIIAr & 1/at &.$/ M A a$ & محيط جغرافيايع & عولمل محيطى & حالات سازمائي \\
\hline رتبه اول & $\%$ r 9 & - Mutraf & 1/49 &.$/ \pi T$ & محيط جغرافيايى & & \\
\hline رتبه دوم & $\% W$ & ./uliva & V/ar & EYT/. & محيط اقتصادى & & \\
\hline
\end{tabular}

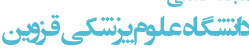

همجئين نتايج برؤهش باليدواسكايت نشان ميدهد عوامل

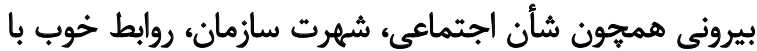

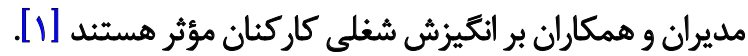
در اين بُروهش منظور از محيط اقتصادى، دو شاخص رونق

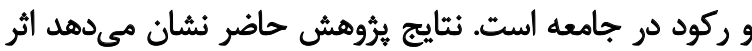
تعديل كنيند

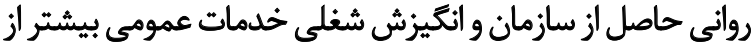

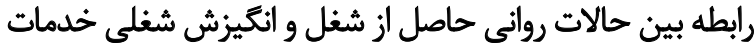

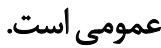

بررسى نقش تعديل كنيدىى عامل محيطى در رابطه بين

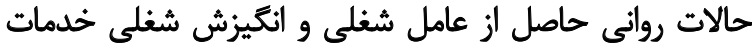

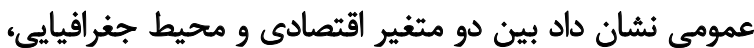

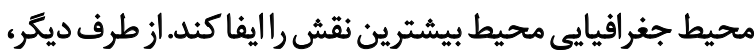

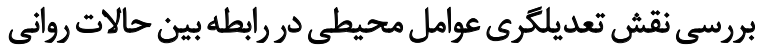

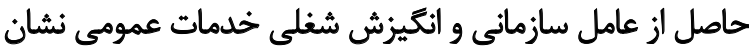
داد محيط اقتصادى بيشترين نقش رازئ رايفا مى كندي

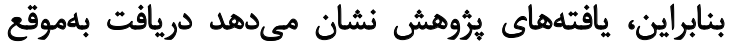

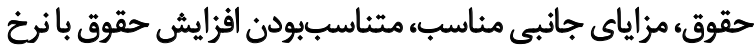

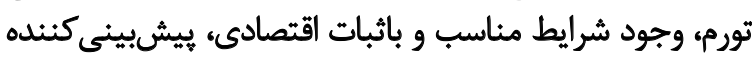

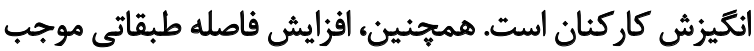

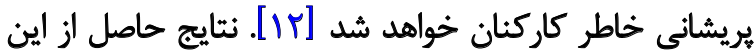

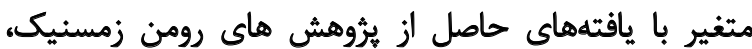

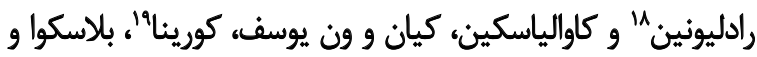

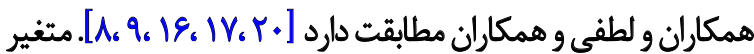

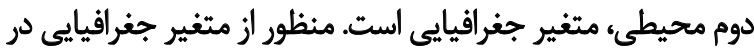

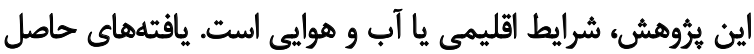

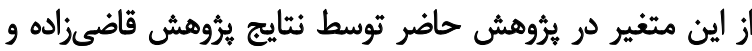

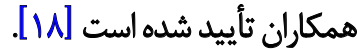
تفاوت اين يثوهش با ديكر يروهشها در اين حوزه، توجه به

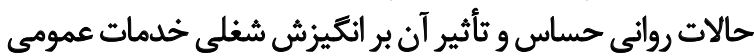

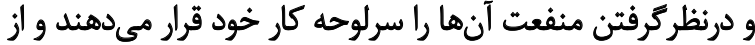

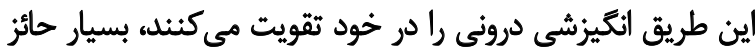

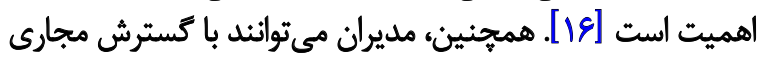

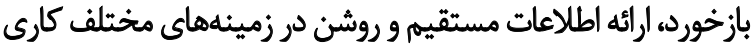

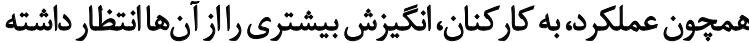

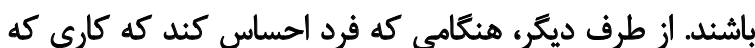

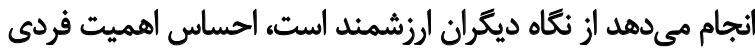

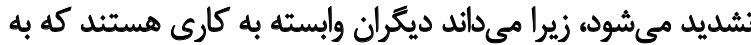

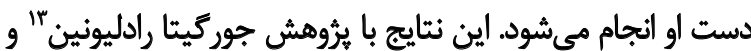

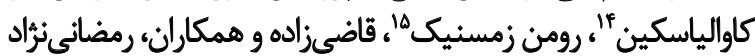

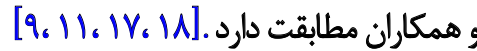

حالات روانى حساس حاصل از عامل سازمانى است كهاين متغيرها

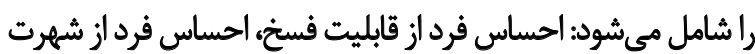
(نمود خارجى) سازمان واحساس فرد از عضويت در سازمان فان (انتخاب).

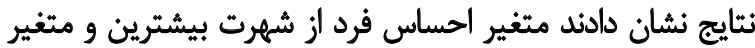

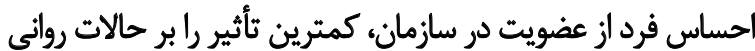

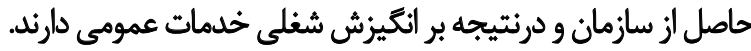

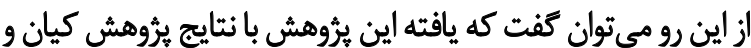

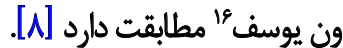

هنغامى كه فرد بتواند درباره نترانى هاى كارى يا مشكلاتى كه

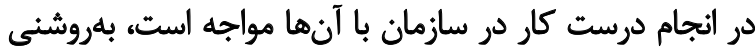

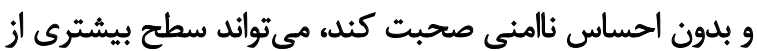

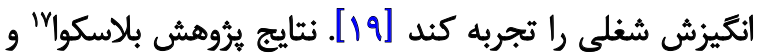

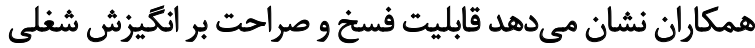

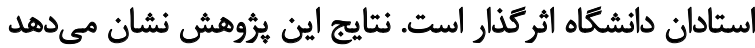

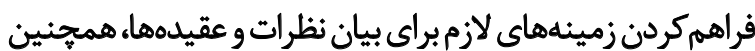

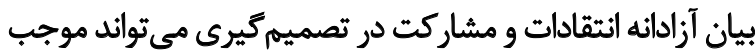

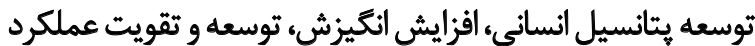

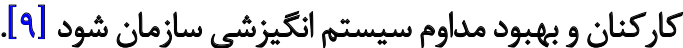

13. Raudeliûniené

14. Kavaliauskiené

15. Zemsnic

16. Kian \& Wan Yusoff

17. Blaskova 
به عنوان يك ييشنياز است. بنابراين اين نكثه خود به بهن عنوان

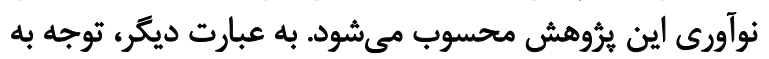

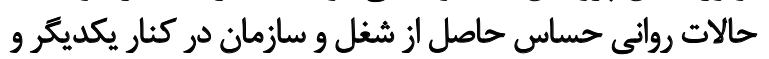

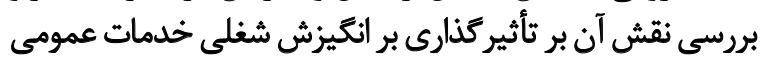

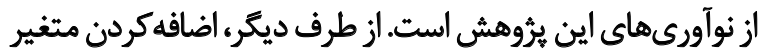

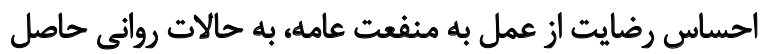

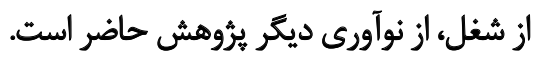

مالامنات أخالاقي

$$
\text { بيروى ازز اصول اخلاق يثوهش }
$$

ملاحظه اخلاقى در يُروهشهاى علوم انسانى و بلويرُه در

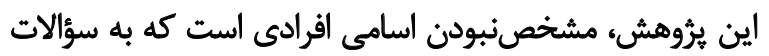
بروهش ياسخ دادهاند.

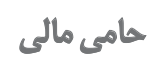

اين مقاله، بركرفته از پاياننامه دكتراى سيده سعيده جوانمرد

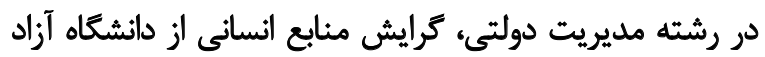

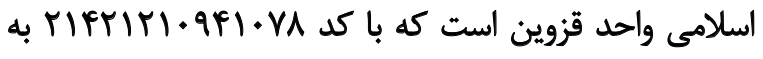
تصويب رسيده است

$$
\begin{aligned}
& \text { اين يرُوهش حامى مالى ندارد. } \\
& \text { هشاركت نويسئدكًان }
\end{aligned}
$$

مفهومسازى، روششناسى، نتارش و و تحليل دادها،

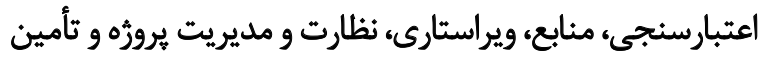

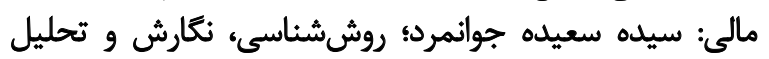

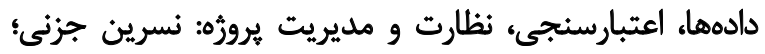

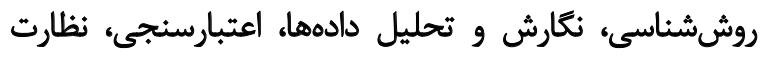

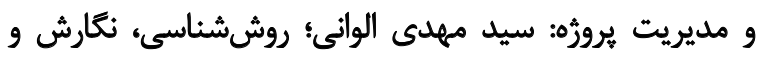

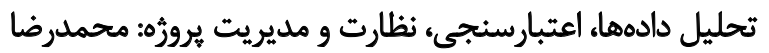
حميدىزاده. تعارض مناقع طبق نظر نويسندكان در اين مقاله تعارض منافع وجود ندارد. 


\section{References}

[1] Palidauskaitè J, Vaisvalavičiūtè A. Analysis of Public Servants' Working in Municipality Administration Motivation Research. Pub policy Adm. 2011; 10(1):127-40. [DOI:10.5755/j01. ppaa.10.1.234]

[2] Ganta VC. Motivation in the workplace to improve the employee performance. Int J Eng Technol, Manage Appl Sci. 2014; 2(6):221-30.

[3] Khoshnevis $\mathrm{H}$, Tahmasebi A. The motivation system in a governmental organization. Procedia-Social Behav Sci. 2016; 230:212-8. [DOI:10.1016/j.sbspro.2016.09.027]

[4] Vandenabeele W. Toward a public administration theory of public service motivation: An institutional approach. Public Manage Rev. 2007; 9(4):545-56. [DOI:10.1080/14719030701726697]

[5] Moorhead G, Griffin R.W. Organizational Behavior [Gh Memarzade, M Alvani, Persian Trans.]. Tehran: Morvarid; 2007.

[6] Recepoğlu E. Analyzing job motivation level of high school teachers in Turkey. Procedia-Social Behav Sci. 2014; 116:22205. [DOI:10.1016/j.sbspro.2014.01.547]

[7] Raeissi P, Mohebbifar R. Job motivator from the employees \& managers' point of view in teaching hospitals affiliated to Qazvin University of Medical Sciences. J Qazvin Uni Med Sci. 2006; 10(1):101-8. [In Persian]

[8] Kian TS, Yusoff WF. Motivation and promotion opportunity of academic citizens towards open innovation: Proposed model. Procedia-Social Behav Sci. 2015; 204:29-35. [DOI:10.1016/j. sbspro.2015.08.106]

[9] Raudeliūnienè J, Meidutè-Kavaliauskienè I. Analysis of factors motivating human resources in public sector. Procedia-Social Behav Sci. 2014; 110:719-26. [DOI:10.1016/j.sbspro.2013.12.916]

[10] Salancik GR, Pfeffer J. A social information processing approach to job attitudes and task design. Administrative Science Quarterly. 1978; 23(2):224-53. [DOI:10.2307/2392563] [PMID]

[11] Ramezani Nejad R, Hemmati Nejad M, Shafii Sh, Ghelichzade M, Loghmani M. Study of the characteristics of a physical education school based on the JCM model. Physiol Manag Stud sports. 2013; 6(2):63-79. [In Persian]

[12] Palidauskaite J. Uniqueness of Public Service Motivation. Public Policy Adm. 2007; 1(19):33-45.

[13] Palidauskaite J, Segalovičienè I. The profile of public servants motivation in Lithuania: Analysis of empirical research results. Management of Organizations: Systematic Research. 2008; 47:73-90

[14] Pedersen MJ. Public service motivation and attraction to public versus private sector employment: Academic field of study as moderator? Int Public Manage J. 2013; 16(3):357-85. [DOI:10.1080/10967494.2013.825484]

[15] Hackman JR, Oldham GR. Development of the job diagnostic survey. J Appl Psychol. 1975; 60(2):159-70. [DOI:10.1037/ h0076546]
[16] Van Loon NM. The role of public service motivation in performance: Examining the potentials and pitfalls through an institutional approach [PhD. dissertation]. Utrecht: Utrecht University; 2015.

[17] Zámečník R. The measurement of employee motivation by using multi-factor statistical analysis. Procedia-Social Behav Sci. 2014; 109:851-7. [DOI:10.1016/j.sbspro.2013.12.553]

[18] Ghazizade M, Amani j. Investigating Factors Affecting Personnel Survival. First National Industrial Management Conference. Shahed University 2016.

[19] Immanuel I, Mas'ud F. [Analisis pengaruh budaya organisasi dan motivasi kerja terhadap kinerja karyawan (Indonesian)]. Diponegoro J Manag. 2017; 6(4):445-55. [DOI:10.1016/S22125671(14)00730-8]

[20] . Lotfi E, Darvishi F, Shafii E, Khodadadi R. Investigating the role of economic, social, and welfare factors in the status of employees of the police. Hum Resour Dev Support. 2013; 9(31): 89-106. [In Persian] 
This Page Intentionally Left Blank 